\title{
Reducing The Computational Requirements for Simulating Tunnel Fires by Combining Multiscale Modelling and Multiple Processor Calculation
}

\author{
Izabella Vermesi ${ }^{\mathrm{a}, 1, *}$, Guillermo Rein ${ }^{\mathrm{b}}$, Francesco Colella ${ }^{\mathrm{c}}$, Morten Valkvist ${ }^{\mathrm{d}}$, Grunde \\ Jomaas $^{\text {a }}$ \\ ${ }^{a}$ Department of Civil Engineering, Technical University of Denmark, 2800 Kongens Lyngby, DK \\ ${ }^{b}$ Department of Mechanical Engineering, Imperial College London, SW7 $2 A Z$ London, UK \\ ${ }^{c}$ Exponent Inc., 9 Strathmore Rd, Natick, MA, USA \\ ${ }^{d}$ Greater Copenhagen Fire Department, Bag Rådhuset 3, 1550 Copenhagen W, DK
}

\begin{abstract}
Multiscale modeling of tunnel fires that uses a coupled 3D (fire area) and 1D (the rest of the tunnel) model is seen as the solution to the numerical problem of the large domains associated with long tunnels. The present study demonstrates the feasibility of the implementation of this method in FDS version 6.0, a widely used fire-specific, open source CFD software. Furthermore, it compares the reduction in simulation time given by multiscale modelling with the one given by the use of multiple processor calculation. This was done using a $1200 \mathrm{~m}$ long tunnel with a rectangular cross-section as a demonstration case. The multiscale implementation consisted of placing a $30 \mathrm{MW}$ fire in the center of a $400 \mathrm{~m}$ long 3D domain, along with two $400 \mathrm{~m}$ long 1D ducts on each side of it, that were again bounded by two nodes each. A fixed volume flow was defined in the upstream duct and the two models were coupled directly. The feasibility analysis showed a difference of only $2 \%$ in temperature results from the published reference work that was performed with Ansys Fluent [1]. The reduction in simulation time was significantly larger when using multiscale modelling than when performing multiple processor calculation (97\% faster when using a single mesh and multiscale modelling; only $46 \%$ faster when using the full tunnel and multiple meshes). In summary, it was found that multiscale modelling with FDS v.6.0 is feasible, and the combination of multiple meshes and multiscale modelling was established as the most efficient method for reduction of the calculation times while still maintaining accurate results. Still, some unphysical flow oscillations were predicted by FDS v.6.0 and such results must be treated carefully.
\end{abstract}

Keywords: CFD, multiscale modelling, tunnel fires, FDS6, computational efficiency

\footnotetext{
*Corresponding author. Tel.: +447763 373681

Email address: i.vermesi140imperial.ac.uk (Izabella Vermesi)

${ }^{1}$ Currently at the Department of Mechanical Engineering, Imperial College London

Preprint submitted to Tunneling and Underground Space Technology
}

December 4, 2016

(c) 2015. This manuscript version is made available under the Elsevier user license

http://www.elsevier.com/open-access/userlicense/1.0/ 


\section{Introduction}

Fire modelling is frequently used as a means of investigating a variety of fire scenarios. Whereas such modelling is feasible for most type of structures, long tunnels are problematic from a numerical point of view, because they, due to their length, are defined by large domains that require very large computational resources. This numerical challenge is amplified by the fact that finding the proper fire safety strategy often requires trying a number of scenarios to establish all the essential characteristics of the system. Among the models found in literature, Vega et al.[2] required $50 \mathrm{~h}$ to simulate $10 \mathrm{~min}$ of a fire using a 3D model in ANSYS Fluent. Other models use either short tunnels [3] or simplified one-dimensional models for longer tunnels [4]. As an alternative to sacrificing either complexity or time efficiency, multiscale modelling for tunnel flows and fires has previously been studied using a general purpose computational fluid dynamics (CFD) software and it has yielded satisfactory results in comparison to full scale CFD simulations [1]. The method combines a 3D domain for the near fire zones, which are characterized by large temperature and pressure gradients, with a 1D network approach for the bulk flow in the far field. A full description of the concept, as well as an assessment of the method's accuracy, is presented by Colella et al. [1], [5]. The

model used in Colella's work has been validated using experimental measurements from real tunnel flows [6]. As the previous study used ANSYS Fluent, there is a need for a feasibility study using other modelling techniques.

Herein, the primary investigation is thus the feasibility of multiscale modelling of tunnel fires in Fire Dynamics Simulator (FDS v.6.0), which is a CFD model for fire driven flow that is widely used for heat and smoke transport from fires [3]. It is worth noting the difference in the governing equations in ANSYS Fluent and FDS: the former uses Reynoldsaveraged Navier Stokes equations (RANS) for its simulations, whereas the latter uses Large Eddy Simulations. A comprehensive analysis of the differences between RANS and LES modelling are found in the work by Versteeg and Malalasekera [7]. Furthermore, FDS is an open-source software, thus it is very widely used, especially in the industry. Therefore, developing a feasible multiscale model for this software can make a significant impact on reducing simulation times for a large number of users. Parametric studies are needed in order to obtain the relevant scenario that is going to be analyzed in detail for the final tunnel ventilation design. This is possibly performed with more computationally heavy programs or settings. As such, the fast computation enables the designer to eliminate several scenarios in the process towards the final design.

The implementation of the multiscale model in FDS v.6.0, sketched in Fig. 1, followed the work by Colella et al. [1], while taking advantage of the fire-specific capabilities of FDS v.6.0. The geometry and model guidelines such as the domain length and volumetric flow induced by the ventilation system served as a start for the present model. The 3D component was created using the traditional 3D grid, while the $1 \mathrm{D}$ network was implemented using the Heating, Ventilation and Air Conditioning (HVAC) feature of FDS v.6.0, which is described in detail in the next section. These two models are coupled directly in the FDS 


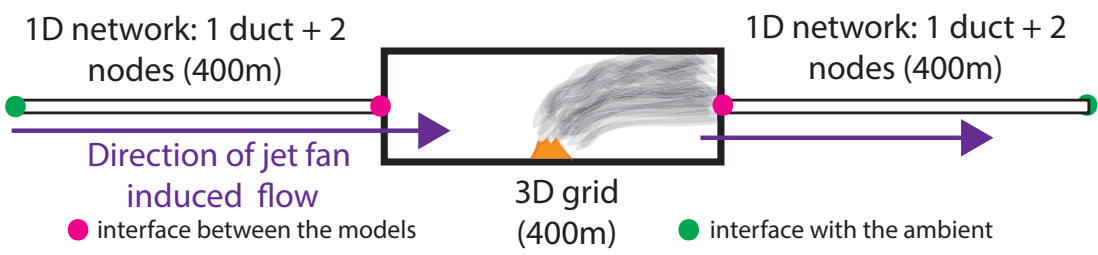

Figure 1: The concept of multiscale modelling of tunnels fires in FDS v.6.0

code, something which provides a continuous interaction between them. This is in contrast with an indirect coupling that requires the use of an additional software to model the 1D network, whose results are then used as input for the boundary conditions of the 3D model. As a result of this direct coupling, the time spent preparing the model is reduced. The coupling of 3D and HVAC component in FDS has been validated against real tunnel flow data in Ang et al. [8], thus confirming the possibility of coupling the two domains.

The second objective of this paper is to assess which of the following methods that is the most time-efficient: multiscale modelling, multiple processor calculations or a combination of the two. Because of the computational requirements, the $3 \mathrm{D}$ grid was divided in multiple meshes assigned to individual cores of the computer. In this way, the order of magnitude of the duration of the simulation decreased from weeks to hours. To find out which method had contributed the most to the decrease in runtime duration, simulations were performed on the same model, first using a single mesh in the 3D domain of the multiscale model to compare with the multiscale-multiple-mesh model. Then the full tunnel was simulated with a single mesh and multiple meshes.

\section{Methodology}

\subsection{D Model and Fire Scenario}

In order to be able to compare the results of the method implemented in FDS with the results obtained in the reference work [1], the tunnel chosen for the analysis has a total length of $1200 \mathrm{~m}$ and a longitudinal ventilation system. It is considered to be a road tunnel with traffic going in one direction only. In the previous work, the tunnel cross-section had a horseshoe shape with a height of $6.5 \mathrm{~m}$ and a cross-sectional area of $53 \mathrm{~m}^{2}$. However, FDS has some constraints regarding geometry and can only contain models with rectangular grids. An attempt was made to simulate a circular cross section using a stair-stepped boundary condition. However this is not a correct solution as it offers a different behavior of the flow, which is not necessarily a realistic behavior. Therefore, an equivalent, rectangular cross-section was used in the current study. In order to obtain an area similar to the previous one, the width was chosen to be $8 \mathrm{~m}$, giving a cross-sectional area of $52 \mathrm{~m}^{2}$. The hydraulic diameter is found to be $D_{H}=\frac{4 A}{P}=7.17 \mathrm{~m}$. The walls, floor and surface were 
beverages with such sensory attributes (Drewnowski \& Gomez-Carneros, 2000; Lesschaeve \& Noble, 2005). Running parallel with supply and demand, highly colored, dark-pigmented superfruits and berries offering potential health benefits often have several constituents that can suffer chemical polymerization and/or degradation during processing, which might augment the already perceived bitterness or astringency (Lawless, Threlfall, Meullenet, \& Howard, 2013). Consumer dissatisfaction of flavor seems especially true in several shelf-stable and/or new to the market flash-pasteurized juices and smoothies containing açaí, blackberry, black cherry, blackcurrant, wild blueberry, cranberry, tart cherry, mangosteen, maqui, and pomegranate (Sabbe, Verbeke, Deliza, Matta, \& Van Damme, 2009; Lawless et al., 2013). Therefore, the long-term increased demand and full potential use of superfruits and their expanding markets may not be sustained if flavor changes and/or possible off-flavors associated with processing, bitterness and astringency are not studied, understood, and ultimately resolved. For example, a product development survey indicated that demand for superfruit beverages may have already lost preference to traditional fruit flavors, and fusion/coupled flavors (Sloan, 2011). Although anecdotally, current world-wide pomegranate market offerings do not seem to support this supposition. A plausible research and development effort for currently produced superfruits and $100 \%$ juices could be improving the existing consumer quality attributes, while maintaining high phytonutrient status.

Most published pomegranate juice articles have not addressed flavor/sensory issues revolving around heating and chemical oxidation (e.g., possible Maillard or Strecker reactions). To the best of our knowledge, only one study to date has used stored commercial organic pomegranate juice in blends and this was presumably a pasteurized product (Vazquez-Araujo, Chambers IV, Adhikari, \& Carbonell-Barrachina, 2010). Four articles have reported sensory and 


\subsubsection{Grid Sensitivity Analysis}

As seen in previous studies, the results given by FDS are highly dependent on the grid size [11]. Reducing the grid size does not automatically mean a significantly better precision, but it does considerably increase the runtime of the simulation. Therefore, it is important to find a balance between the desired precision and keeping the simulation time at a level acceptable to the user.

It is important to state that the grid cells in FDS are recommended to have a cubic shape [12]. This is possible in the current case, thus each cell has the same width, length and height. According to the User's Guide for FDS [12], an initial sizing of the mesh cells should be done by evaluating the non-dimensional expression $D^{*} / \partial x$ where $\partial x$ is the cell size and $D^{*}$ is the characteristic fire diameter. For the desired HRR of $30 \mathrm{MW}, D^{*}=3.7$ in this case, cell sizes should fall between $0.23 \mathrm{~m}$ and $0.92 \mathrm{~m}$, according to results from previous studies [12]. Therefore, a cell size of $0.40 \mathrm{~m}$ is chosen as an initial setting. As the range above is just a guideline, a mesh sensitivity analysis was performed for mesh sizes of $0.20 \mathrm{~m}, 0.25 \mathrm{~m}$ and $0.80 \mathrm{~m}$.

The results for temperature and longitudinal velocity, further called u-velocity, were recorded at $210 \mathrm{~m}$, which represent a distance of $10 \mathrm{~m}$ from the center of the fire source. Recording devices were placed every $0.5 \mathrm{~m}$ from a height of $0.5 \mathrm{~m}$ until $6 \mathrm{~m}$ in order to obtain a more complete overview of the fire behavior. Fig. 3 shows the average results over time for temperature and velocity for each of the cell sizes used. $10 \mathrm{~m}$ away from the fire, the grid using $0.80 \mathrm{~m}$ cells gives higher results for the temperature values, but the $0.40 \mathrm{~m}, 0.25 \mathrm{~m}$ and $0.20 \mathrm{~m}$ cell grid show similar results. The difference between the $0.40 \mathrm{~m}$ and the $0.25 \mathrm{~m}$ cell grid is not significant, meaning that decreasing the cell size does not necessarily induce a considerable increase in the precision. Also, the results from the coarse mesh are quite stable compared with the results from the moderate and finer meshes, which have some oscillations in the mass flow due to a numerical issue in the solver. The velocity results at both distances follow similar trends. The coarse mesh predicts lower values of the flow velocity, whereas the other ones are similar. The average results are presented in Table 1, along with the variation from the results of the $0.20 \mathrm{~m}$ mesh. The biggest difference is, as expected, in the mesh with cell size of $0.80 \mathrm{~m}$. The $0.40 \mathrm{~m}$ and $0.25 \mathrm{~m}$ meshes do not differ much from the finest mesh, with none of the average values having differences of more than $6 \%$.

Therefore, in the case of temperatures, the errors are negligible, with a variation of only a few degrees that would not influence the overall fire safety design. As for the velocity, the average values for the $0.20 \mathrm{~m}, 0.25 \mathrm{~m}$ and $0.40 \mathrm{~m}$ meshes are almost identical at $210 \mathrm{~m}$ and very similar at $300 \mathrm{~m}$.

The above results show that there is no significant precision improvement in decreasing the cell size from $0.40 \mathrm{~m}$. The differences are negligible for the performance of the fire safety design. However, the differences in runtimes are very significant. As shown in Table 1, 


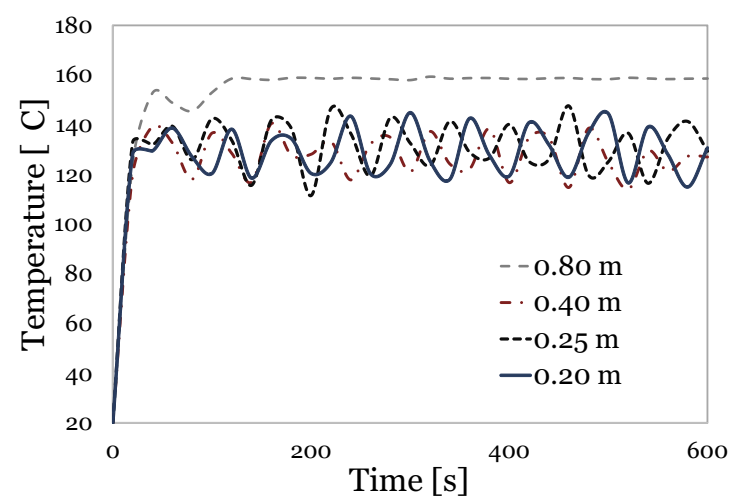

(a) Temperature

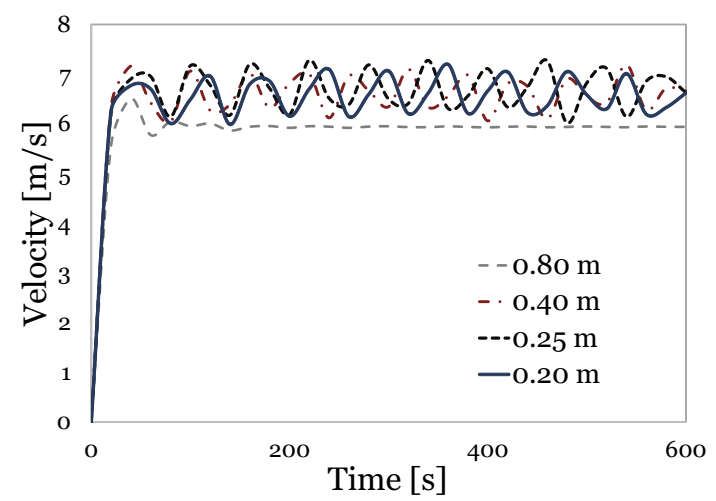

(b) U-velocity

Figure 3: Grid sensitivity analysis: the average temperature (a) and the average u-velocity (b) results for 4 different cell size configurations

there is a very important increase in computational demand by decreasing the cell size. The difference in the number of cells is reflected in the number of hours required for the calculations. Calculations with the finest mesh take takes $92 \mathrm{~h}$, whereas calculations with the 0.40 $\mathrm{m}$ mesh are about 15 times faster. As the difference in precision does not justify using the finest mesh, the $0.40 \mathrm{~m}$ cell mesh was chosen for the remainder of the study.

Table 1: Comparison of the average results from simulations with different cell sizes, including runtime

\begin{tabular}{lllll}
\hline & $0.20 \mathrm{~m}$ & $0.25 \mathrm{~m}$ & $0.40 \mathrm{~m}$ & $0.80 \mathrm{~m}$ \\
\hline Average temperature at $210 \mathrm{~m}\left[{ }^{\circ} \mathrm{C}\right]$ & 126 & 128 & 124 & 152 \\
Deviation & - & $+2 \%$ & $-1 \%$ & $+21 \%$ \\
\hline Average temperature at $300 \mathrm{~m}\left[{ }^{\circ} \mathrm{C}\right]$ & 114 & 118 & 112 & 110 \\
Deviation & - & $+4 \%$ & $-2 \%$ & $-3 \%$ \\
\hline Average velocity at $210 \mathrm{~m}[\mathrm{~m} / \mathrm{s}]$ & 6.6 & 6.7 & 6.6 & 5.9 \\
Deviation & - & $+2 \%$ & $+1 \%$ & $-10 \%$ \\
\hline Average velocity at $300 \mathrm{~m}[\mathrm{~m} / \mathrm{s}]$ & 7.4 & 7.3 & 7.0 & 6.5 \\
Deviation & - & $0 \%$ & $-6 \%$ & $-12 \%$ \\
\hline Runtime $[\mathrm{h}]$ & 92 & 39 & 6 & 0.8 \\
\hline
\end{tabular}




\subsubsection{Multiple Meshes}

FDS uses MPI (Message-Passing Interface) to allow multiple computers to run a single FDS simulation [12]. The FDS domain is divided into multiple meshes with equal cell sizes that are then computed as different processes. This approach has previously been used to produce satisfactory results for tunnel fire simulations [13].

To ensure that the mesh division did not add significant errors to the results of the simulation, a comparison was done using a single mesh and 3 meshes, respectively. The domain was divided into 3 separate meshes, which were then assigned to individual processes. The measurements were made $0.40 \mathrm{~m}$ away from the boundary interface using temperature and velocity slice files. Fig. 4 shows the velocity and the temperature profiles versus tunnel height as recorded in the location of the boundary interfaces for both the single mesh and the multiple mesh. Because the first boundary interface was positioned upstream of the fire, the temperature information was not analyzed for this position. The velocity profile at the first interface and the temperature profile at the second interface show that the boundary interfaces induce negligible differences from the single mesh model. The velocity at the second interface has the same profile and the values differ with a maximum of $0.2 \mathrm{~m} / \mathrm{s}$. As a result, it was deemed appropriate to use multiple processes in the feasibility calculations, and the grid was divided further, resulting in 17 meshes.

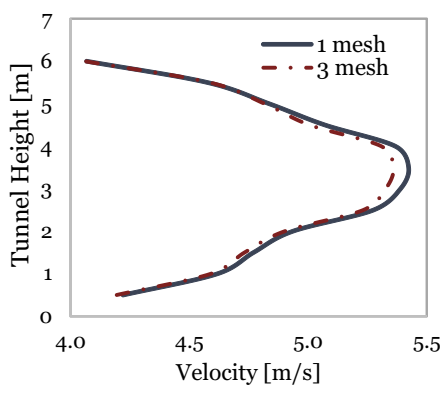

(a) U-velocity

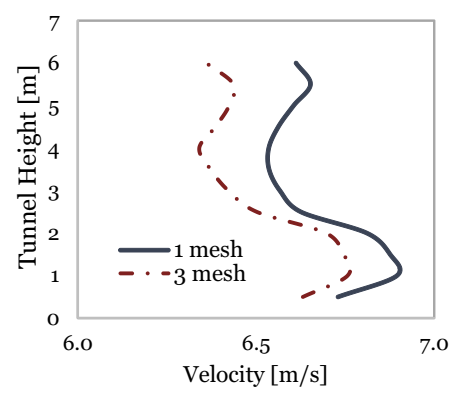

(b) U-velocity

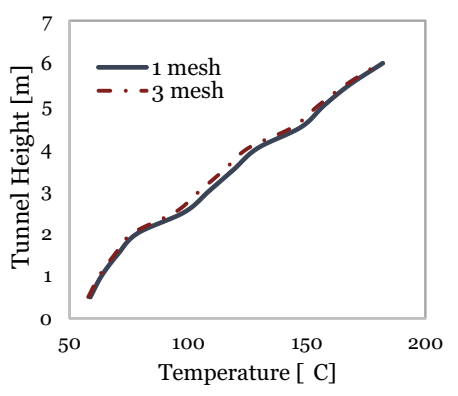

(c) Temperature

Figure 4: Comparison of results for the 1 mesh scenario with the 3 mesh scenario at the first (a) and second $(b, c)$ measurement interfaces, placed at $0.40 \mathrm{~m}$ away from the boundary interfaces

\subsection{D Model and Multiscale Coupling}

The Heating, Ventilation and Air Conditioning (HVAC) module has been added to FDS in order to provide coupling between the solutions of the conservation equations for inlets and outlets of a HVAC network and the computational grid [14]. It is a 1D model consisting of a network of ducts and nodes, where the former represents any continuous flow path without node interruption and the latter represents the point where the duct joins the FDS computational domain, the ambient or where multiple ducts intersect, and thus form a junction 
[15]. This model uses an explicit solver for the conservation equations of mass and energy along with an implicit solver for the conservation equation of momentum. There is a caveat of the boundary conditions of the ducts, as no thermal loss takes place through the ducts. However, as this mainly influences the chimney effect in inclined tunnels, the impact on the no-slope tunnel used herein is negligible.

In order to simulate the rest of the $1200 \mathrm{~m}$ long tunnel, the duct and node network was defined as follows. On each side of the FDS grid, a node was placed at the interface with the FDS grid and the other one at $400 \mathrm{~m}$ distance. The duct connecting them has an area equal to the cross-sectional area of the tunnel, thus $52 \mathrm{~m}^{2}$, and a friction coefficient of 0.026 , as recommended by Jang and Chen [16]. This yields a relative roughness of 0.003 according the the Moody chart (for tunnels with wind speeds between 1 and $6.5 \mathrm{~m} / \mathrm{s}$ ). From the relative roughness, the absolute roughness, which is the input parameter, is obtained and has a value of $\mathrm{k}=0.024 \mathrm{~m}$.

The ventilation system considered in the fire scenarios consists of 5 pairs of fans at the upstream portal. The pairs of jet fans on the south side of the tunnel are considered not to be operational. This ventilation scenario is used for comparison with scenario 2 from Colella et al. [1]. In order to specify the equivalent flow of 5 pairs of fans into an equivalent fan defined in the duct, a value of mass flow rate of $300 \mathrm{~kg} / \mathrm{s}$ was taken from the full-scale CFD results for scenario 2 [1]. This value was obtained in the reference cases built for the validation of multiscale modelling [1], cases that serve as a reference point for this work. For the sake of calculation simplicity, the fan was defined as having a constant volume flow. However, this way of imposing a flow condition does not capture the throttling effect of the fire on the ventilation velocity and it does not take into account the surrounding pressure field [14]. The throttling effect is the tendency of the fire to resist the airflow [17], which means that the same ventilation scenario cannot be used for fire sizes ranging from small to large and without causing differences in the flow response. However, considering that the aim of this study is to assess the feasibility of implementing multiscale modelling in FDS v.6, not to design a viable ventilation scenario for a tunnel, using a constant flow is deemed acceptable.

The information exchange between the two models is illustrated in Fig. 5. The initial step is to update the density solution, which is followed by the calculation of the average values (temperature, pressure, species) at each HVAC node coupled to the main grid gas phase solution. The next step is to perform the equations for the HVAC solvers, then use this solution to update the boundary conditions. By updating the divergence, the cycle continues and the calculation moves on to the next time step. 


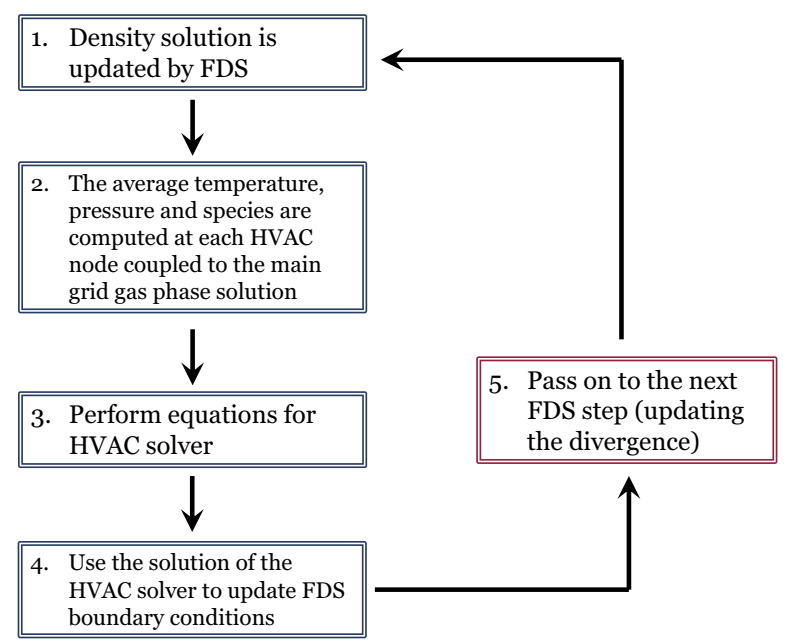

Figure 5: Solving procedure for HVAC + FDS model

\section{Results and Discussion}

\subsection{Multiscale Modelling Feasibility in FDS v.6.0}

As temperatures and velocities are the most important parameters, it is of interest to see how they compare to the results obtained in the reference work. However, it is important to establish a few of the differences between the two models that arise from the different modelling techniques.

The FDS model was designed to investigate fire flows [12] and has a dedicated combustion model. This simulates the fire behavior differently from the volumetric heat source used to model the fire in Fluent, which is a general CFD tool that can be used for a variety of situations. The behavior of the fire in FDS is influenced by the buoyancy forces as well as by the rate at which the fire is 'fed' oxygen by the ventilation system.

Furthermore, there is a difference in the way the two programs handle meshes. Fluent uses an unstructured grid to be able to simulate more realistic geometrical shapes, whereas FDS uses a structured, rectangular grid, and, as such, simplifies the geometry to be able to perform the calculation on rectangular cells. As Colella et al. [1] used Reynolds-Average Navier-Stokes equations, they could use a finer mesh in Fluent without requiring a computational effort as great as FDS would require to solve the same mesh. As FDS uses the large eddy simulation method, it does not average the flow, thus requiring more time for calculations. Also, Colella et al. [1] used a steady state simulation, while the simulation in FDS is transient and captures the fire for 10 minutes. Thus, it is obvious that the results, although averaged, will have some discrepancies.

Taking into account these differences, the longitudinal flow conditions in the tunnel are presented in Fig. 6 and 7. It is clear that the flow becomes fully-developed with only small 
differences in temperature and velocity across the cross-section as it approaches the interface with the 1D model. The temperature and velocity results were evaluated at 10 and $100 \mathrm{~m}$ from the center of the fire. As seen in Fig. 8 and 9, the flow $10 \mathrm{~m}$ downstream of the fire presents a clear division between the area of the plume and the rest of the tunnel. The area around the plume has a much higher velocity than the plume itself, due to the ventilation air bypassing the plume on its sides. Further away from the fire, at $100 \mathrm{~m}$, the flow becomes more homogeneous, with a higher overall velocity. This confirms the assumption that the flow develops enough to be modelled as a one-dimensional flow. The centerline time-averaged results for a section of the 3D domain that compare the FDS and ANSYS models are shown in Fig. 10 and 11. The shape of the temperature distribution has similar characteristics and the values are very similar, thus proving that multiscale modelling works as well for FDS as it did for ANSYS Fluent. However, it must be noted that the velocity in this scenario is much higher than the reference values because of the way the flow is fixed. If a quadratic fan curve were to be implemented, the throttling effect of the fire would interact with the fan flow and the values of the velocity would be reduced.

\subsection{Reduction in Computational Resources}

The most significant advantage of multiscale modelling is reducing the duration of simulations. As multiple processor calculations have the same purpose, it is interesting to see which of these two methods is more efficient. To make a comparison, four simulations were made: one with the full tunnel using a single mesh, one with the full tunnel using multiple meshes, one with multiscale modelling with a single mesh and one with multiscale modelling combined with multiple meshes. The model was the same as the one used in the feasibility analysis. The jet fans, which were implemented as a fixed flow in the multiscale model, were modeled as simple vents with a volume flow.

For a comparison regarding simulation times, it is important to specify the computational resources that were available. The simulations were performed on a single computer with $2.20 \mathrm{GHz}$ CPU clock and 18 cores. Because of the centrally placed fire, the model had to be divided into an odd number of meshes. Therefore, 17 meshes were used for the multiple meshes simulations.

Table 2 shows the runtimes of each analyzed model. Using a single mesh and modelling the entire tunnel length of $1200 \mathrm{~m}$ resulted in a simulation that required an estimated 3 weeks for completion, which is not practical when having to investigate many scenarios. The estimation was calculated using the total required wall clock time as the ratio between the elapsed wall clock time and elapsed FDS simulation time multiplied by the total FDS simulation time [14]. Trying to overcome this issue by dividing the grid into multiple meshes halved the runtime, but it is still impractically long. However, using a multiscale model reduced the runtime to less than a day, which makes it viable for design purposes. If multiple processors are available, combining multiple meshes and multiscale modelling results in the most time-saving solution. The drawback of multiple processor calculation is obviously the 
dependence on the available number of processors, a problem which does not appear when using multiscale modelling. This method reduced the simulation time from weeks to less than a day and can thus be applied on a variety of computer configurations.

Table 2: Comparison between the runtimes using multiscale modelling and multiple processor calculation

\begin{tabular}{ll}
\hline Tunnel model & Runtime $[\mathrm{h}]$ \\
\hline Full tunnel: single mesh & 519 (est.) \\
Full tunnel: multiple (17) mesh & 280 (est.) \\
Multiscale model (3D length of 400m): single mesh & 13.2 \\
Multiscale model (3D length of 400m): multiple (17) mesh & 6.1 \\
\hline
\end{tabular}

\subsection{Validation with Experiments and Oscillatory Mass Flows}

In order to validate the multiscale model with experiments, a comparison of cold-flow conditions between the simulation of the tunnel and experimental measurements was carried out and discussed by Ang et al. [8]. That work compares measurements of mass flow through the Dartford tunnel with a full CFD model and a multiscale model done in FDS v.6.1. It is shown that average velocities are very similar in the full CFD and multiscale models and that they compare well with the measurements. The model presented in the current work formulates the concept for the model used by the authors of the validation [8]. Therefore, the validation is applied for the multiscale model methodology using FDS v.6.0. However, there were no experimental measurements of an actual fire in the Dartford tunnel to serve as validation, so the validation in the cold flow scenario was used.

As shown by Ang et al. [18], when adding a fire load to the multiscale model in FDS v.6.1, the mass flows show an oscillating behavior. This can be observed in the results of the current work as well, which was done in FDS v.6.0, and are worthy of consideration because they signify a numerical issue within the solver that might influence the results.

As shown in Fig. 3, this oscillatory mass flow influences both centerline temperatures and velocities for meshes with medium or fine cell size,but are not appearing in the mesh with large cell size $(0.8 \mathrm{~m})$. Instead of reaching steady-state, the mass flow rate varies in time following a periodic oscillations. This is a numerical issue encountered in the solver of both FDS v.6.0 and 6.1 and has to be investigated in the newer versions of FDS, which have been released since the publication of this work.

The oscillatory mass flow is an issue of multiscale modelling in FDS v.6.0 and 6.1, regardless of the way the ventilation flow is modelled. This work uses a simple constant mass flow as a boundary at the 1D-3D interface, whereas Ang [18] uses a 3D domain to model the flow 
coming from the jet fans. This issue is specific to the multiscale model.

\section{Conclusions}

Based upon the current feasibility analysis, multiscale modelling is suitable for FDS v.6.0 using the HVAC component. The temperature results show good agreement with the reference work values, displaying a difference of just $2 \%$. The differences in the velocity results arise from the difference in flow definition between the two models (FDS and ANSYS Fluent). The fixed flow used herein (FDS) induces a much larger flow velocity than the quadratic flow used in the reference work (ANSYS Fluent), and as a result the velocity is much higher in the current case.

When implemented in FDS v.6.0, multiscale modelling provides the fastest way to simulate a large number of fire scenarios. It does not depend on the computational resources as much as the multiple processor calculation does. It also reduces the simulation time significantly while keeping the precision of the results. Compared to the original full-scale tunnel with a single mesh, multiscale modelling using a single mesh reduced the simulation duration by 97 $\%$, so a simulation took a few hours instead of weeks. As a comparison, a multiple processor calculation where the model was divided into 17 meshes only resulted in a $46 \%$ reduction in computational time. If possible, the most efficient way is to combine both methods, and in the current study a $99 \%$ reduction in the necessary computational time was obtained for this combination. Of course, these values are subject to the available computing resources. However, the ranking between them is independent of the computational power.

It is important to specify that the purpose of this work was to assess the feasibility of multiscale modelling in FDS v.6.0 and compare its simulation time reduction to the one given by using multiple processor calculation. The study was not aimed at analyzing the efficiency of a particular fire safety design strategy. For such considerations, flow conditions have to be improved. For the future work, defining a quadratic flow using a fan curve is extremely important in order to capitalize on the interaction between the 3D model and the 1D HVAC network. In addition, the oscillatory mass flows that result from a numerical issue have to be checked in the latest version of FDS.

Keeping in mind the oscillations and the lack of validation in fire conditions, this method can be cautiously adopted to look at steady state conditions near the fire. The information can be used in the design of the tunnel structure, as well as in establishing the passive fire protection (PFP) measures to be used in the fire safety design of the tunnel. The heat losses can be accurately modelled in the 3D domain of the model. Also, the 3D domain is large enough to contain the smoke, thus the potential issue of defining the walls of the tunnel as adiabatic is acceptable, since it provides a more conservative estimates. 


\section{Acknowledgements}

The authors would like to thank NIST and VTT for providing the FDS v.6.0 code as open-source.

\section{References}

[1] F. Colella, G. Rein, R. Borchiellini, J. L. Torero, A Novel Multiscale Methodology for Simulating Tunnel Ventilation Flows During Fires, Fire Technology 47 (1) (2010) 221-253.

[2] M. Vega, K. Diaz, J. Oro, R. Tajadura, C. Morros, Numerical 3D simulation of a longitudinal ventilation system: Memorial Tunnel case, Tunnelling and Underground Space Technology 23 (2008) 539-551.

[3] S. Jain, S. Kumar, S. Kumar, T. Sharma, Numerical simulation of fire in a tunnel: Comparative study of CFAST and CFX predictions, Tunnelling and Underground Space Technology 23 (2008) 160-170.

[4] E. Migoya, A. Crespo, J. Garcia, J. Hernandez, A simplified model of fires in road tunnels. Comparison with three-dimensional models and full-scale measurements, Tunnelling and Underground Space Technology 24 (2009) 37-52.

[5] F. Colella, G. Rein, V. Verda, R. Borchiellini, Multiscale modeling of transient flows from fire and ventilation in long tunnels, Computers \& Fluids 51 (1) (2011) 16-29.

[6] F. Colella, G. Rein, R. Carvel, P. Reszka, J. L. Torero, Analysis of the ventilation systems in the Dartford tunnels using a multi-scale modelling approach, Tunnelling and Underground Space Technology 25 (2010) 423-432.

[7] H. Versteeg, W. Malalasekera, An Introduction to Computational Fluid Dynamics: The Finite Volume Method, Prentice Hall, 2007.

[8] C. Ang, G. Rein, J. Peiro, R. Harrison, Simulating longitudinal ventilation flows in long tunnels: Comparison of full CFD and multi-scale modelling approaches in FDS6, Tunnelling and Underground Space Technology 52 (2016) 119-126.

[9] F. Colella, G. Rein, R. Borchiellini, V. Verda, One-dimensional and multi-scale modelling of tunnel ventilation and fires, in: Handbook of Tunnel Fire Safety, 2012, pp. 365-390.

[10] R. Carvel, A. Beard, The Handbook of Tunnel Fire Safety, Thomas Telford Publishing, 2005.

[11] N. Petterson, Assessing the feasability of reducing the grid resolution in fds field modeling, Tech. rep., University of Canterbury (2002).

[12] J. Floyd, G. Forney, S. Hostikka, T. Korhonen, R. McDermott, K. McGrattan, C. Weinschenk, NIST Special Publication 1019, Fire Dynamics Simulator, User's Guide, sixth Edition, 2013.

[13] P. Weisenpacher, L. Halada, J. Glasa, Computer Simulation of Fire in a Tunnel Using Parallel Version of FDS, in: Seventh Mediterranean Combustion Symposium, Cagliari, 2011.

[14] J. Floyd, G. Forney, S. Hostikka, T. Korhonen, R. McDermott, K. McGrattan, C. Weinschenk, NIST Special Publication 1019, Fire Dynamics Simulator Technical Reference Guide, Volume 1: Mathematical Model, sixth Edition, 2013.

[15] J. Floyd, Coupling a Network HVAC Model to a Computational Fluid Dynamics Model Using Large Eddy Simulation, Fire Safety Science (10) (2011) 459-470.

[16] H.-M. Jang, F. Chen, On the determination of the aerodynamic coefficients of highway tunnels, Journal of Wind Engineering and Industrial Aerodynamics 90 (8) (2002) 869-896.

[17] A. Vaitkevicius, F. Colella, R. Carvel, Investigating the Throttling Effect in Tunnel Fires, Fire Technology (2015) 1-10.

[18] C. Ang, Investigation of a computationally efficient multi-scale modelling method in long tunnels for fire dynamics simulator 6, Master's thesis, Imperial College London (2014). 


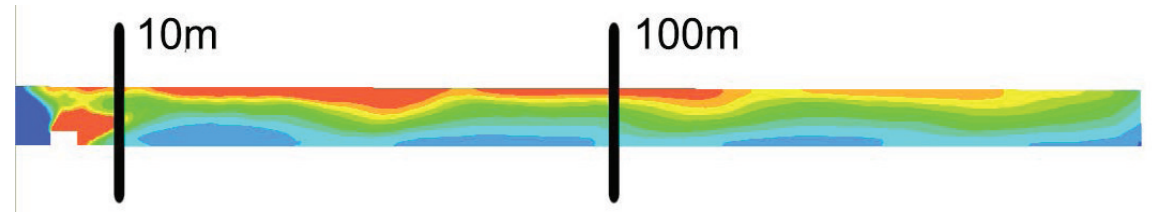

Figure 6: Longitudinal slice of the downstream flow behavior through the tunnel center line: average temperature in ${ }^{\circ} \mathrm{C}$; the fire is represented by the white box on the left of the figure; cross-sectional slices at 10 and $100 \mathrm{~m}$ which present the temperature values are shown in Fig. 8

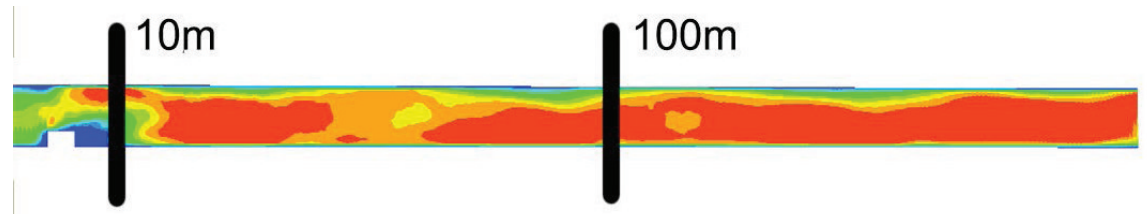

Figure 7: Longitudinal slice of the downstream flow behavior through the tunnel center line: average $\mathrm{u}$-velocity in $\mathrm{m} / \mathrm{s}$; the fire is represented by the white box on the left of the figure; cross-sectional slices at 10 and $100 \mathrm{~m}$ which present the temperature values are shown in Fig. 9

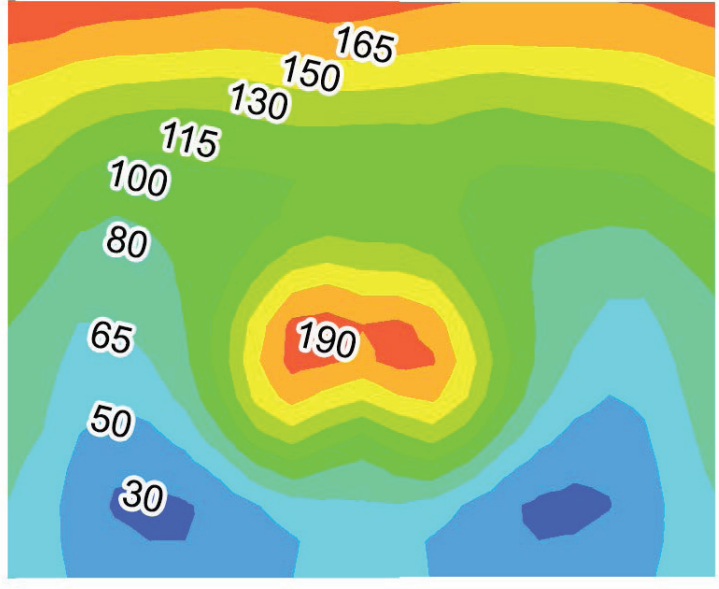

(a) $10 \mathrm{~m}$

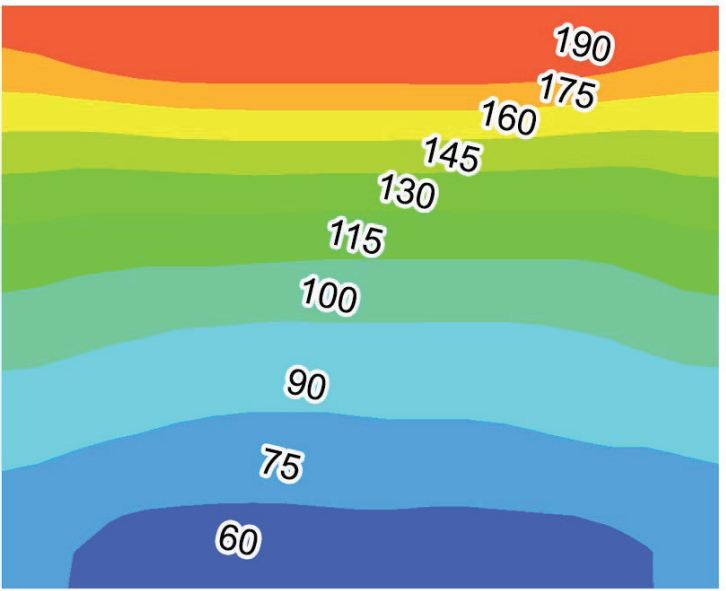

(b) $100 \mathrm{~m}$

Figure 8: Cross-sectional slice of the average temperature $\left(\right.$ in $\left.{ }^{\circ} \mathrm{C}\right)$ at various distances downstream from the fire, as shown in Fig. 6 


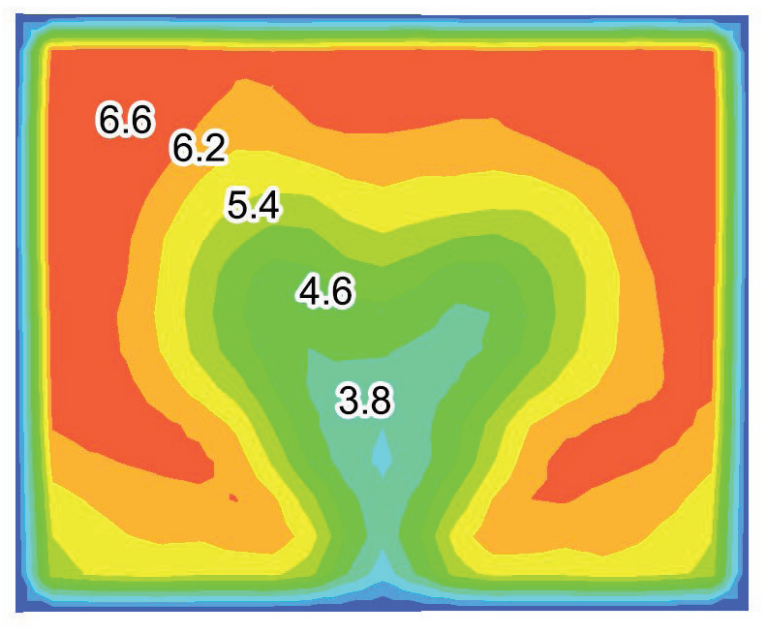

(a) $10 \mathrm{~m}$

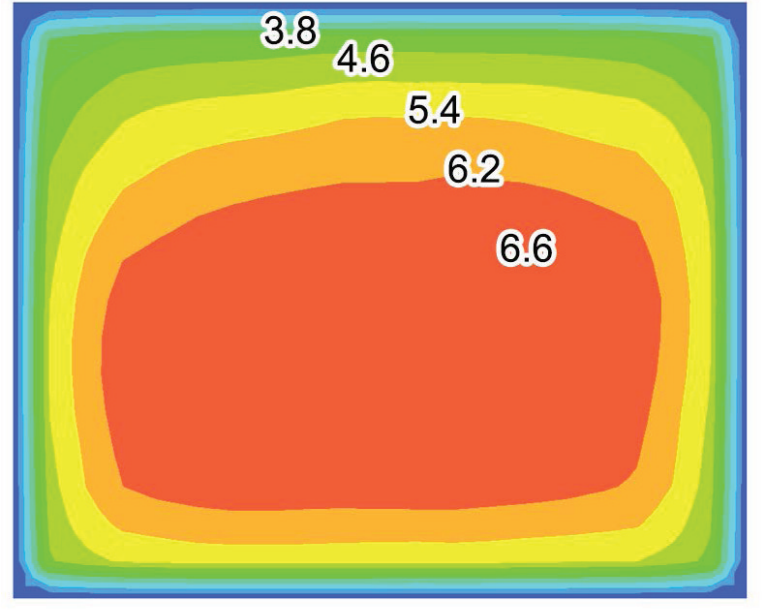

(b) $100 \mathrm{~m}$

Figure 9: Cross-sectional slice of the average $\mathrm{u}$-velocity (in $\mathrm{m} / \mathrm{s}$ ) at various distances downstream from the fire, as shown in Fig. 7
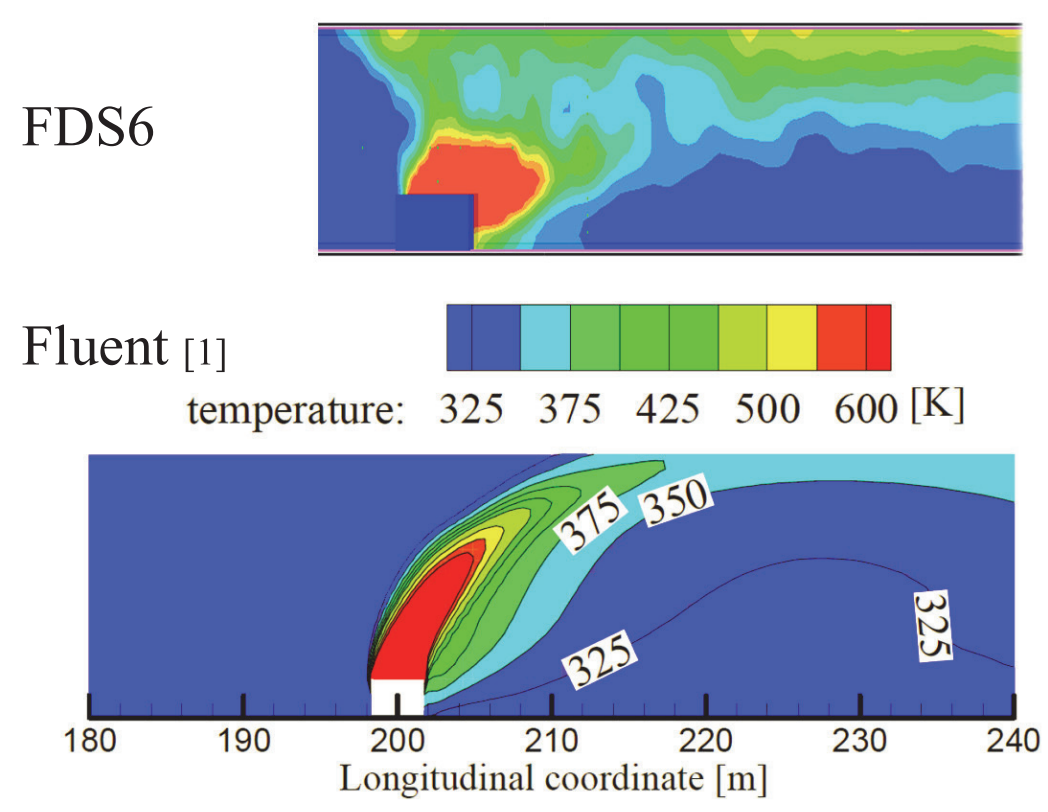

Figure 10: Temperature slice at 600s - 5 working jet fan pairs, 30MW fire, 3D domain of $400 \mathrm{~m}$, cell size of $0.4 \mathrm{~m}$, time-averaged results; the length of the FDS domain is identical to the one in Fluent, with the difference arising from image scaling 


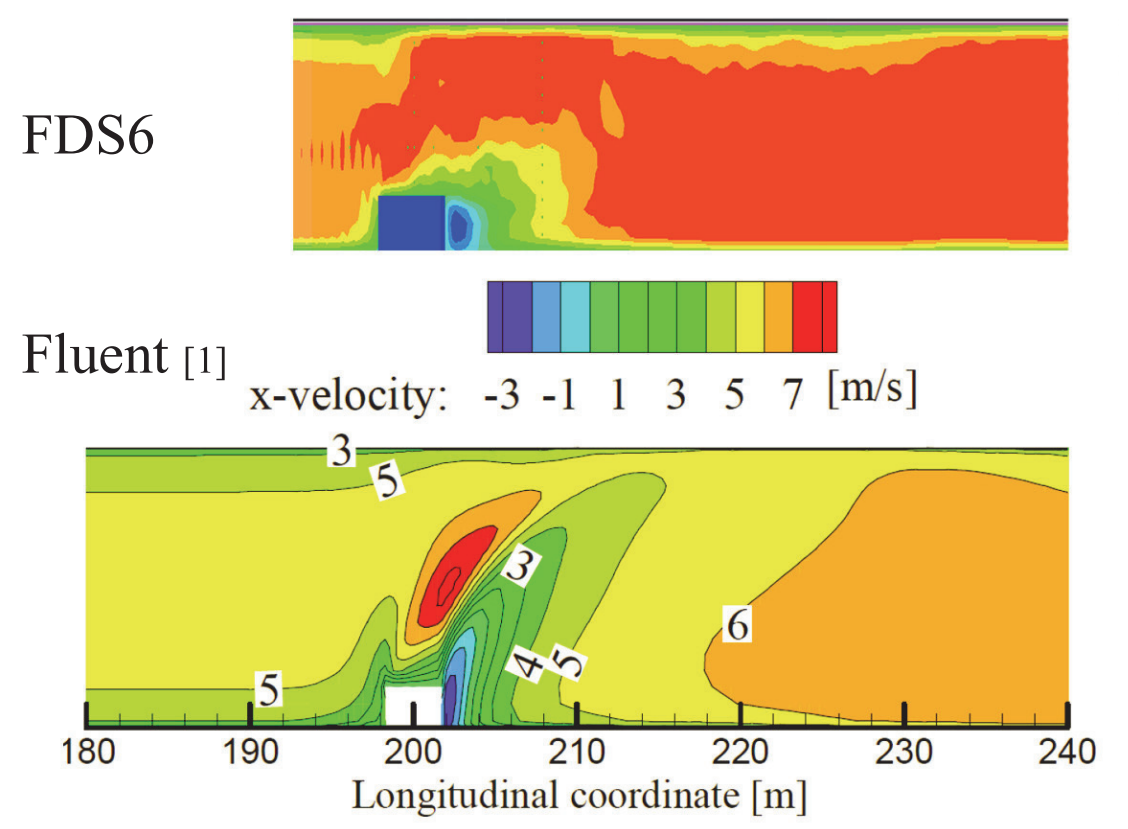

Figure 11: U-velocity (x-velocity) slice at 600s - 5 working jet fan pairs, 30MW fire, 3D domain of $400 \mathrm{~m}$, cell size of $0.4 \mathrm{~m}$, time-averaged results; the length of the FDS domain is identical to the one in Fluent, with the difference arising from image scaling 